\title{
Self-Anticoagulant Nanocomposite Spheres for the Removal of Bilirubin from Whole Blood: A Step towards Wearable Artificial Liver
}

Xin Song, ${ }^{a, ~ \# ~ T a o ~ X u, ~}{ }^{a, ~}{ }^{*}$ Li Yang, ${ }^{b}$ Yupei Li,${ }^{c}$ Ye Yang, ${ }^{a}$ Lunqiang Jin, ${ }^{a}$ Jue Zhang, ${ }^{a}$ Rui Zhong, ${ }^{d}$ Shudong Sun, ${ }^{a}$ Weifeng Zhao, ${ }^{a, e^{*}}$ and Changsheng Zhao ${ }^{a}$

${ }^{\text {a }}$ College of Polymer Science and Engineering, State Key Laboratory of Polymer Materials Engineering, Sichuan University, Chengdu, 610065, People's Republic of China

${ }^{\mathrm{b}}$ Department of Polymer Science and Engineering, Zhejiang University, Hangzhou 310027, People's Republic of China

${ }^{\mathrm{c}}$ Institute for Disaster Management and Reconstruction, Sichuan University, Chengdu 610225, People's Republic of China

${ }^{\mathrm{d}}$ Institute of Blood Transfusion, Chinese Academy of Medical Sciences, Peking Union Medical College, Chengdu 610052, People's Republic of China

${ }^{\text {e }}$ State Key Laboratory of Molecular Engineering of Polymers, Fudan University

*Corresponding author. E-mail: zhaoscukth@163.com; weifeng@scu.edu.cn (Weifeng Zhao)

Tel.: +86-28-85400453; Fax: +86-28-85405402.

${ }^{\#}$ These authors contributed equally to this work. 


\section{Experimental Section}

\section{Materials}

Polyethersulfone (PES, Ultrason E6020P, $\mathrm{M}_{\mathrm{w}}$ 58,000) was obtained from the BASF chemical company. Sodium alginate (SA), acrylamide (AM), N, N'-methylene bisacrylamide (MBA), ammonium persulfate (APS), N, N'-dicyclohexylcarbodiimide (DCC), sodium hydroxide $(\mathrm{NaOH})$, calcium carbonate $\left(\mathrm{CaCO}_{3}, \leqslant 30 \mu \mathrm{m}\right)$, vitamin $\mathrm{C}$ (VC), phosphate-buffered saline (PBS) and bilirubin were purchased from Aladdin Reagent Co. Ltd. Graphite flakes were obtained from Sigma-Aldrich and aqueous graphene oxide (GO) solution was prepared by a modified Hummer's method from the natural graphite flakes. ${ }^{1} \mathrm{~N}, \mathrm{~N}$-Dimethylformamide (DMF), normal saline (0.9 \% $\mathrm{NaCl})$, sulfuric acid $\left(\mathrm{H}_{2} \mathrm{SO}_{4}\right)$, dichloromethane $\left(\mathrm{CH}_{2} \mathrm{Cl}_{2}\right), \mathrm{N}, \mathrm{N}$ '-dimethylacetamide (DMAc) were purchased from Chengdu Kelong Chemical Reagent Co. Ltd. (China). Bovine serum albumin (BSA) and bovine fibrinogen (BFG) were obtained from Sigma Chemical Co. Ltd. Activated partial thromboplastin time (APTT) reagent, prothrombin time (PT) reagent, thrombin time (TT) reagent, coagulation factor V deficient plasma, coagulation factor VIII deficient plasma, coagulation factor IX deficient plasma, coagulation factor XI deficient plasma, coagulation factor XII deficient plasma and antithrombin III reagent were purchased from SIEMENS Co. Ltd. Micro BCA ${ }^{\mathrm{TM}}$ Protein Assay Reagent kit was purchased from PIERCE Inc. Human complement fragment 3a (C3a) kit, human complement fragment 5a (C5a) kit, human platelet factor4 (PF4) kit, and human bradykinin (BK) kit were purchased from Cusabio Biotech, China. Thrombin antithrombin III complex (TAT) kit was purchased from 
Enzygnost TAT micro, Assay Pro, USA. Deionized (DI) water was prepared from a Millipore purification system (Billerica, MA, USA) and used during the whole study. All the chemicals were used as received.

\section{Characterization of HepMBm and rGO@HepMBm}

For Fourier transform infrared spectroscopy (FTIR) analysis, sodium alginate (SA), HepMBm, rGO@HepMBm0, rGO@HepMBm1, rGO@HepMBm2 and rGO@HepMBm3 were completely dried, and then a FTIR spectrometer (Nicolet 560, USA) was used to obtain the FTIR spectra between 500 and $4000 \mathrm{~cm}^{-1}$, using the $\mathrm{KBr}$ disk method. The thermogravimetric analysis (TGA) curves of SA, HepMBm, rGO@HepMBm0,rGO@HepMBm1,rGO@HepMBm2 and rGO@HepMBm3 were obtained by using a Q500 thermogravimetric analyzer (TG209F1, Netzsch, Germany) under a dry nitrogen atmosphere from 30 to $800{ }^{\circ} \mathrm{C}$ at the heating speed of $10{ }^{\circ} \mathrm{C} / \mathrm{min}$. The curves of derivative thermogravimetric analysis were derived from TGA data. Nuclear magnetic resonance $\left({ }^{1} \mathrm{H}\right.$ NMR) spectra of SA and HepMBm were performed by using a $400 \mathrm{MHz}$ NMR spectrometer (Bruker AV II, Switzerland) and $\mathrm{D}_{2} \mathrm{O}$ used as solvent. Element analysis (EA) data of SA, HepMBm and heparin were collected via an organic carbon/elemental carbon analyser (Atmoslytic DRI2001A, USA), and used for calculating the degree of substitution (i.e., sulfonic acid groups per uronic acid on the backbone of HepMBm). Scanning electron microscopy and energy dispersive spectra (EDS) were investigated using a JMS-7500F SEM (JEOL, Japan) to obtain the cross-section and surface morphologies and the elemental compositions of the rGO@HepMBm0, rGO@HepMBm1, rGO@HepMBm2 and rGO@HepMBm3. To 
prepare SEM samples, the spheres were cut off after being immersed in liquid nitrogen for 1 minute and then completely freeze-dried overnight. Afterwards, the spheres were attached to a support and then coated with a gold layer under vacuum. Finally, the SEM observation was carried out at an accelerating voltage of $5 \mathrm{kV}$.

The swelling ratios of the rGO@HepMBm0, rGO@HepMBm1, rGO@HepMBm2 and rGO@HepMBm3 were analyzed via a gravimetric method. ${ }^{2}$ A certain number of the spheres were dried in an oven at $60{ }^{\circ} \mathrm{C}$ for more than 2 days to get a constant weight. Then the dried spheres were re-immersed into 5 different liquid conditions including DI water, normal saline, PBS, platelet poor plasma (PPP) and whole blood, respectively. Afterward, the wet spheres were weighted at different times after gently removing excess solution with a filter paper. The swelling ratios of the spheres were calculated by using the following equation:

$$
\text { Swelling ratio }(g / g)=\frac{W_{w^{-}} W_{d}}{W_{d}}
$$

where $W_{\mathrm{w}}(\mathrm{g})$ is the constant weight of the spheres at wet states; $W_{\mathrm{d}}(\mathrm{g})$ is the weight of the spheres at dried states.

To investigate the mechanical properties of the spheres, rGO@HepMBm0, rGO@HepMBm1, rGO@HepMBm2 and rGO@HepMBm3 were thoroughly swollen in PBS and then applied to a universal mechanical testing machine (SANS CMT4000) with a constant speed of $2 \mathrm{~mm} /$ minute under a $200 \mathrm{~kg}$ load mechanical sensor.

\section{Hemocompatibility}

\subsection{Plasma collection}

Healthy human fresh blood (from three 24-year-old male donors) was collected using 
vacuum tubes (5 mL, Jiangsu Kangjian Inc., China) containing sodium citrate or ethylene diamine tetraacetic acid as anticoagulant (anticoagulant-to-blood ratio, 1:9 $(\mathrm{v} / \mathrm{v}))$. For plasma collection, the blood was centrifuged at $1000 \mathrm{rpm}$ for 15 minutes to obtain platelet-rich plasma (PRP) or at $4000 \mathrm{rpm}$ for 15 minutes to obtain platelet-poor plasma (PPP). The same donor blood samples were used all through the blood tests.

The experiments were approved and performed by West China Hospital, Sichuan University, and all the experiments were performed in compliance with the relevant laws and national guidelines (GB/T 16886.4-2003/ISO 10993-4:2002, General Administration of Quality Supervision, Inspection and Quarantine of the People's Republic of China, Standardization Administration of the People's Republic of China). Informed consent was obtained for any experimentation with human subjects, and all regulations (e.g. IRB) were fulfilled for using human blood.

\subsection{Clotting times}

Activated partial thromboplastin time (APTT) and thrombin time (TT) were measured to investigate the anticoagulant properties of the HepMBm; while APTT, TT and prothrombin time (PT) were utilized to investigate the anticoagulant properties of the rGO@HepMBm0,rGO@HepMBm1, rGO@HepMBm2 and rGO@HepMBm3. The tests were performed by a semiautomatic blood coagulation analyzer CA-50 (Sysmex Corporation, Kobe, Japan), and the process was as follows: the samples were pre-immersed in PBS overnight and then incubated at $37^{\circ} \mathrm{C}$ for $1 \mathrm{~h}$. After that, the PBS was removed, and $200 \mu \mathrm{L}$ of fresh PPP was introduced. After incubating at $37{ }^{\circ} \mathrm{C}$ for $30 \mathrm{~min}, 50 \mu \mathrm{L}$ of the incubated PPP was moved to a test cup and mixed with $50 \mu \mathrm{L}$ of 
APTT agent (Dade Actin Activated Cephaloplastin Reagent, Siemens; incubated 10 min at $37{ }^{\circ} \mathrm{C}$ before use), followed with adding $50 \mu \mathrm{L}$ of $0.025 \mathrm{M} \mathrm{CaCl}_{2}$ solution, and then the APTT was measured. For the TT test, $50 \mu \mathrm{L}$ of the incubated PPP was added in a test cup and mixed with $100 \mu \mathrm{L}$ of thrombin agent (Sysmex; incubated 10 min at $37{ }^{\circ} \mathrm{C}$ before use), and then the TT was measured. For the PT test, $50 \mu \mathrm{L}$ of the incubated PPP was added in a test cup, followed by the addition of $100 \mathrm{ml}$ Thromborel $\mathrm{S}$ (Siemens; incubated $10 \mathrm{~min}$ before use), and incubated at $37{ }^{\circ} \mathrm{C}$ for $2 \mathrm{~min}$, and then the PT was measured.

\subsection{Plasma recalcification tests}

Plasma recalcification tests of the spheres were performed to investigate the anticoagulant activities of the spheres in real blood environment. The testing process was as follows: The spheres were pre-immersed in PBS overnight and then incubated at $37^{\circ} \mathrm{C}$ for 1 hour. After that, the PBS was removed, and $300 \mu \mathrm{L}$ of fresh PPP was introduced. After incubating at $37{ }^{\circ} \mathrm{C}$ for 30 minutes, clotting was initiated by recalcification of PPP with $6 \mu \mathrm{L} 5$ wt. $\% \mathrm{CaCl}_{2}$ under continuously oscillation. The plasma solution was monitored for clotting by manually dipping of a stainless-steel hook coated with silicone into the solution to detect fibrin threads. Then the plasma recalcification time (PRT) was recorded at the first sign of fibrin formation. The plasma was considered as non-coagulation when the PRT was over 180 minutes. Coagulation was analyzed for every 30 seconds between the two readings to increase accuracy and the digital photos of plasma incubated with different spheres after recalcification were recorded. 


\subsection{Coagulation factor activity and antithrombin III activity}

For the detection of the activities of factor V, VIII, IX, XI, XII and antithrombin III, in plasma, $5 \mu \mathrm{L}$ of the incubated PPP was added in a test cup and diluted with $45 \mu \mathrm{L}$ buffer solution. After incubating at $37{ }^{\circ} \mathrm{C}$ for 30 seconds, the complex solution was mixed with $50 \mu \mathrm{L}$ corresponding factor deficient plasma (Coagulation Factor $\mathrm{V}$ deficient plasma, Coagulation Factor VIII deficient plasma, Coagulation Factor IX deficient plasma, Coagulation Factor XI deficient plasma and Coagulation Factor XII deficient plasma; Sysmex; incubated 10 minutes at $37{ }^{\circ} \mathrm{C}$ before use). After another 30 second incubation at $37^{\circ} \mathrm{C}, 50 \mu \mathrm{L}$ of APTT agent was added, followed with adding 50 $\mu \mathrm{L}$ of $0.025 \mathrm{M} \mathrm{CaCl}_{2}$ solution after incubating at $37{ }^{\circ} \mathrm{C}$ for 30 minutes, and then the factor activity was measured. The antithrombin-activating activity of the spheres were investigated using antithrombin III activity assay kit (Siemens).

\subsection{Hemolysis analysis}

The hemolysis test was carried out to evaluate the erythrocyte compatibility of the spheres. $5 \mathrm{~mL}$ of whole blood was firstly added to $10 \mathrm{~mL}$ of calcium- and magnesiumfree PBS solution, and then the red blood cells (RBCs) were isolated from plasma by centrifuging at $2000 \mathrm{rpm}$ for $10 \mathrm{~min}$, the centrifugation procedure was repeated at least 5 times. For the hemolysis test, $1 \mathrm{~mL}$ of the diluted RBC suspension (approx. 108 cells per $\mathrm{mL}$ ) was added to the spheres (a certain amount of the spheres was previously immersed in PBS overnight) and incubated at $37^{\circ} \mathrm{C}$ for $3 \mathrm{~h}$. PBS was selected as negative control while DI water was used as positive control. Then the suspensions were centrifuged at $8000 \mathrm{rpm}$ for $3 \mathrm{~min}$, and the absorbance of the released hemoglobin 
in the suspensions was measured at $540 \mathrm{~nm}$ using a UV-vis spectrometer (UV-1750, Shimadzu Co., Ltd, Japan), and then the hemolysis ratios of the spheres could be calculated by the following formula:

$$
\text { Hemolysis ratio }(\%)=\frac{A_{s}-A_{n}}{A_{p}-A_{n}} \times 100
$$

where $A_{s}$ is the absorbance of the suspensions, $A_{p}$ and $A_{n}$ are the absorbance the positive control and the negative control, respectively.

\subsection{Evaluation of platelet activation}

The evaluation of platelet activation was measured via an enzyme-linked immune sorbent assay with a Human Platelet Factor4 (PF4) kit (Cusabio Biotech, China). The spheres were pre-immersed in PBS in a 24-well cell culture plate overnight. Then, the PBS was removed and $250 \mu \mathrm{L}$ of human whole blood was introduced. After being incubated at $37^{\circ} \mathrm{C}$ for 1 hour, the whole blood was centrifuged for 10 minutes at 2500 g centrifugal force $\left(2-8^{\circ} \mathrm{C}\right)$ to obtain plasma. Then $40 \mu \mathrm{L}$ of the obtained plasma was diluted for 10 times with PF4-Sample Diluent; then $200 \mu \mathrm{L}$ of the diluted plasma was added into another Antibody Coated Well (provided by the PF4 kit). Finally, the detections were conducted according to the respective instruction manuals. Whole blood was used as control sample.

\subsection{Blood count assay in vitro}

Blood count assays were performed to investigate the effect of the spheres on blood cells. Firstly, a certain amount of spheres were pre-immersed in $200 \mu \mathrm{L}$ of PBS (pH=7.4) in a 48-well culture plate overnight and equilibrated at $37{ }^{\circ} \mathrm{C}$ for 1 hour. Secondly, 200 $\mu \mathrm{L}$ of fresh whole blood from the volunteer was introduced into each well after the 
removal of PBS. Thirdly, the spheres were incubated with whole blood at $37^{\circ} \mathrm{C}$ for 60 minutes and then the rest of the blood were collected. The whole blood cell differential counts were measured by an automated haematology cell analyzer (BC-5100, Mindray Bio-Medical Electronics Co., Ltd., Shenzhen, China) under the provided instruction manuals.

\subsection{Evaluation of complement activation}

Commercial enzyme-linked immune sorbent assays (ELISA) were employed to evaluate the complement activation (Human Complement Fragment 3a (C3a) and Human Complement Fragment 5a (C5a), Cusabio Biotech, China) of the spheres. The spheres were pre-immersed in PBS in a 24-well cell culture plate overnight. Then, the PBS was removed and $250 \mu \mathrm{L}$ of human whole blood was introduced. After being incubated at $37^{\circ} \mathrm{C}$ for 1 hour, the whole blood was centrifuged for 10 minutes at 2500 g centrifugal force $\left(2-8^{\circ} \mathrm{C}\right)$ to obtain plasma. For the $\mathrm{C} 3 \mathrm{a}$ test, $5 \mu \mathrm{L}$ of the obtained plasma was diluted for 500 times with C3a-Sample Diluent, and $100 \mu \mathrm{L}$ of the diluted plasma was added into an Antibody Coated Well (provided by C3a kit). For the C5a test, $10 \mu \mathrm{L}$ of the obtained plasma was diluted for 10 times with C5a-Sample Diluent, and the diluted plasma was added into another Antibody Coated Well (provided by C5a kit). Finally, the detections were conducted according to the respective instruction manuals. Whole blood was used as control sample.

\subsection{Evaluation of activation of contact activation system and kallikrein/kinin}

\section{system}

The evaluation of activation level of contact activation system and kallikrein/kinin 
system for the spheres were measured via an enzyme-linked immune sorbent assay with a Human Thrombin-antithrombin III complex (TAT) kit (Enzygnost TAT micro, Assay Pro, USA) and a Human Bradykinin (BK) kit (Cusabio Biotech, China). The spheres were pre-immersed in PBS in a 24-well cell culture plate overnight. Then, the PBS was removed and $250 \mu \mathrm{L}$ of human whole blood was introduced. After being incubated at $37^{\circ} \mathrm{C}$ for 1 hour, the whole blood was centrifuged for 10 minutes at $2500 \mathrm{~g}$ centrifugal force $\left(2-8{ }^{\circ} \mathrm{C}\right)$ to obtain plasma. For the TAT test, $50 \mu \mathrm{L}$ of the obtained plasma was added into an Antibody Coated Well (provided by the TAT kit). For the BK test, $50 \mu \mathrm{L}$ of the obtained plasma was mixed with $100 \mu \mathrm{L}$ of horse radish peroxidase (HRP)labelled antibody (provided by the BK kit). Finally, the detections were conducted according to the respective instruction manuals. Whole blood was used as control sample.

\subsection{The safety and stability of HepMBm}

Blood count assay was performed to investigate the impact of HepMBm on blood cells. HepMBm was dissolved in whole blood at a concentration of $5 \mu \mathrm{g} / 100 \mu \mathrm{L}$ blood. Then $200 \mu \mathrm{L}$ of normal whole blood and whole blood after dissolving HepMBm were introduced into each well of a 48 -well culture plate. The culture plate was then incubated at $37{ }^{\circ} \mathrm{C}$ for 60 minutes and then the rest of the blood were collected. The whole blood cell differential counts were measured by an automated hematology cell analyzer (BC-5100, Mindray Bio-Medical Electronics Co., Ltd., Shenzhen, China) under the provided instruction manuals. The immunogenicity of HepMBm was evaluated by the activation level of complement activation system, kallikrein activation 
system, platelet activation system and contact activation system. HepMBm was dissolved in whole blood at a concentration of $5 \mu \mathrm{g} / 100 \mu \mathrm{L}$ blood, while normal whole blood was used as control group. Whole blood with or without HepMBm was incubated at $37^{\circ} \mathrm{C}$ for 60 minutes and centrifuged at $1000 \times \mathrm{g}$ for $15 \mathrm{~min}$ to obtain plasma. Then the plasma level of different activation systems was evaluated with a commercial enzyme-linked immune sorbent assay (ELISA) method according to the respective manufacturer's instructions (BD Co., USA). To investigate the stability of the conjugation, 4 nanocomposite spheres were incubated with PBS overnight. Then the UV spectra for pristine PBS and the solution after incubating with nanocomposite spheres were recorded by an UV-vis spectrometer (UV-1750, Shimadzu).

\section{Adsorption experiments}

Firstly, bilirubin powder was dissolved in $2 \mathrm{~mL}$ of $0.1 \mathrm{M} \mathrm{NaOH}$ solution, and the solution was diluted by PBS to a certain concentration. The adsorption experiments were performed in a dark room since bilirubin could be easily degraded as exposed to the light. ${ }^{3}$ To investigate the effect of initial bilirubin concentrations on the adsorption amounts, 4 spheres of each sample were added into bilirubin solutions with different initial concentrations $(100,150,200,250,300,400 \mathrm{mg} / \mathrm{L})$. Then the experiments were performed at a shaking speed of $125 \mathrm{rpm}$ at $37^{\circ} \mathrm{C}$ until adsorption equilibrium. The concentrations of the bilirubin solutions were detected by an UV-vis spectrometer (UV1750, Shimadzu) at $438 \mathrm{~nm}$ and the adsorption amounts of the spheres were determined as follows:

$$
q_{e}=\frac{V\left(C_{0}-C_{e}\right)}{m}
$$


where $q_{e}(\mathrm{mg} / \mathrm{g})$ is the adsorption amount of bilirubin at equilibrium, $C_{0}$ and $C_{e}(\mathrm{mg} / \mathrm{L})$ are the concentrations of the bilirubin solutions before and after adsorption, $V(\mathrm{~L})$ is the volume of the bilirubin solution, $m(\mathrm{mg})$ is the weight of the dried spheres.

To investigate the adsorption kinetics, 4 spheres of each sample were put into glass bottles containing $10 \mathrm{~mL}$ of bilirubin solutions with an initial concentration of $200 \mathrm{mg} / \mathrm{L}$. Then the experiments were performed and the concentrations of the bilirubin solutions at different time intervals $(0,15,45,60,75,90,105,120,150,180,210,270$ and 330 minutes) were calculated as above mentioned.

Plasma proteins adsorption experiments were carried out with bovine serum albumin (BSA) and bovine fibrinogen (BFG) solutions under static condition. Firstly, two spheres were immersed in PBS solution, containing BSA (or BFG) with the concentration of $1 \mathrm{mg} / \mathrm{mL}$, and incubated at $37^{\circ} \mathrm{C}$ for $1 \mathrm{~h}$; then the spheres were rinsed slightly with PBS and DI water. Then the spheres were placed in a washing solution ( $2 \%$ sodium dodecyl sulfate (SDS), $0.05 \mathrm{M} \mathrm{NaOH}$ ) at $37{ }^{\circ} \mathrm{C}$, and shaken for $2 \mathrm{~h}$ to remove the adsorbed protein. The adsorption and desorption times were carefully determined in preliminary experiments. The protein concentration in the washing solution was determined by using the Micro $\mathrm{BCA}^{\mathrm{TM}}$ Protein Assay Reagent Kit (Pierce).

The effect of BSA concentrations on the adsorption amounts for bilirubin was explored in the BSA-rich solution. The initial BSA concentrations were set as 2 and 10 g/L, respectively. 4 spheres of each sample were added into bilirubin solutions (300 $\mathrm{mg} / \mathrm{L}$ ) with different BSA concentrations. Then the experiments were performed until adsorption equilibrium, and the adsorption amounts for bilirubin were calculated as 
above mentioned.

\section{Statistical analysis}

Each experiment was performed independently in duplicate at least, and quantified with at least triplicates. The results are expressed as the mean \pm standard deviation of each sample, as indicated by error bars in all graphs. Statistical analysis was performed using the IBM Statistical Package for the Social Sciences (v.24, SPSS, Chicago, IL, USA). Unpaired Student's t test analysis or one-way analysis of variance (ANOVA) followed by Dunnett's test was used for the statistical analysis of the data. A difference with a $p$ value of less than 0.05 was considered statistically significant. 


\section{Results and discussion:}

The FTIR spectra are shown in Figure S1, a new major peak at $1250 \mathrm{~cm}^{-1}$ and a minor peak at $790 \mathrm{~cm}^{-1}$ were observed in the spectrum of HepMBm comparing with the spectrum of pristine $S A$, which could be attributed to the stretching vibrations of $S=O$ and S-O, respectively. ${ }^{4,5}$ These two new peaks demonstrated the successful sulphation of SA, and the degree of substitution (DS) was high because the absorption peak at $1250 \mathrm{~cm}^{-1}$ was strong. ${ }^{6}$ In addition, a new absorption peak appeared at $1624 \mathrm{~cm}^{-1}$ in the spectrum of HepMBm, which could be attributed to the $\mathrm{C}=\mathrm{O}$ of $-\mathrm{COOH}$ groups. Since DI water was utilized to purify the HepMBm through dialysis and the final $\mathrm{pH}$ was approximately 6-7, part of - $\mathrm{COO}^{-}$might be protonated. While the peak migrated to 1612 $\mathrm{cm}^{-1}$ for the spectrum of $\mathrm{SA}$, and few stretching vibrations of $\mathrm{C}=\mathrm{O}$ could be detected, since the $-\mathrm{COOH}$ groups in pristine SA almost completely existed in the form of deprotonated state. ${ }^{5}$

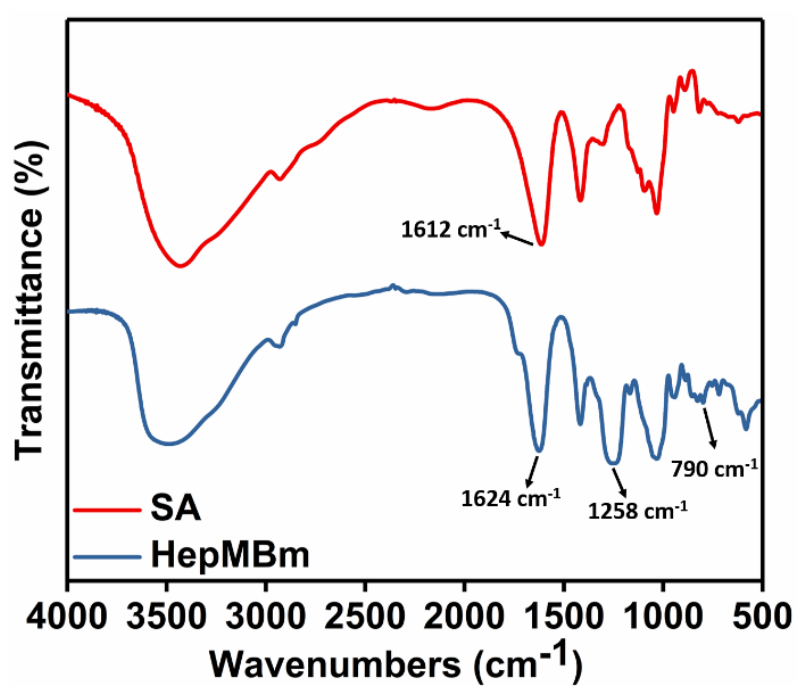

Figure S1. FTIR spectra for SA and HepMBm. 
The ${ }^{1} \mathrm{H}$ NMR for SA and HepMBm are shown in Figure S2. The chemical shifts between 1-1.9 ppm in the spectrum of HepMBm might belong to methylene protons of the residual 1,3-dicyclohexylurea, ${ }^{7,8}$ while the chemical shifts at 2-3 ppm might belong to organic solvents used during the sulphation reaction. The minor chemical shift at 3.3 ppm in the spectrum of SA was assigned to hydroxy protons. However, similar chemical shift was not observed in the spectrum of HepMBm, which was ascribed to the substitution of hydroxyls with sulphate ester groups. Additionally, the chemical shifts between 3.5-4.5 and 5-5.5 which belonged to ring protons and anomeric protons, ${ }^{9}$ respectively, were found to shift to lower field position in the spectrum of HepMBm. This was also attributed to the attachment of electronegative sulphate ester groups.
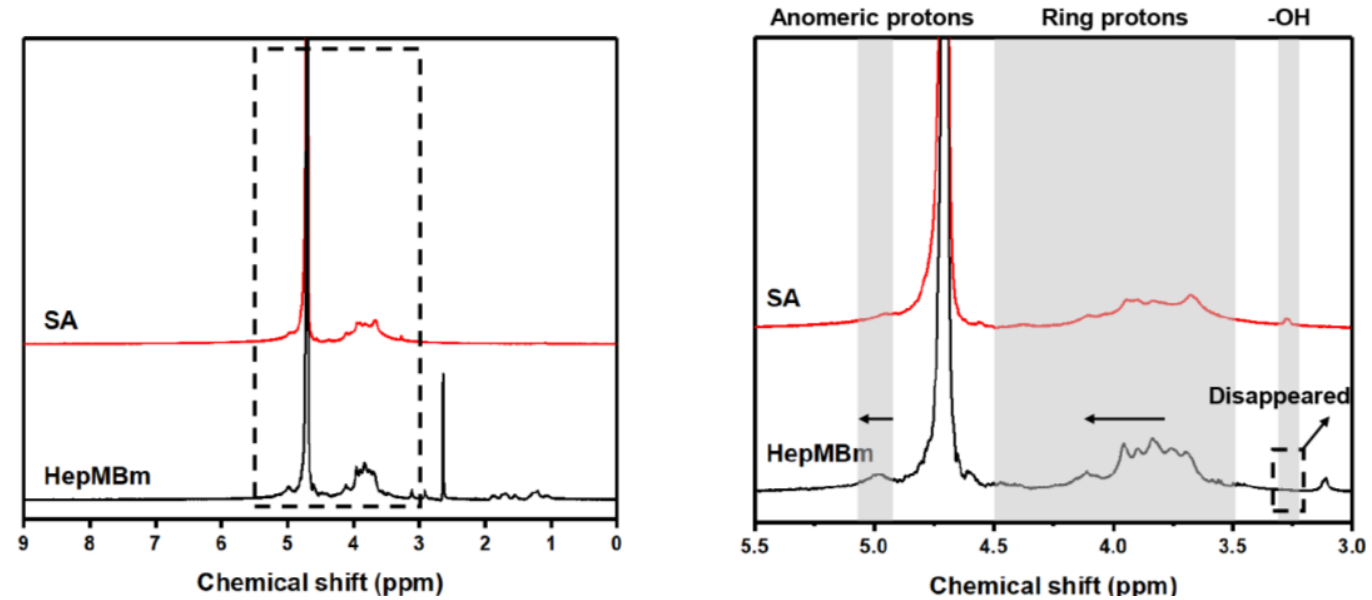

Figure S2. ${ }^{1} \mathrm{H}$ NMR for SA and HepMBm. 
TGA and derivative thermogravimetry (DTG) were utilized to investigate the thermal stabilities of SA and HepMBm, and the results are shown in Figure S3. The TGA curves suggested that the weight loss of pristine SA could be divided into two stages: (i) The vaporization of water molecules. (ii) The thermal degradation of alginate chains. For the TGA curve of HepMBm, the first stage and second stage similar to those of SA were also observed, while a new third stage was exposed, which might be ascribed to the decomposition of $-\mathrm{SO}_{3}{ }^{-}$groups or the residual DCC reagents. Additionally, higher weight retention ratio of HepMBm $(40.9 \%)$ at $700{ }^{\circ} \mathrm{C}$ was observed comparing with that of pristine $\mathrm{SA}(31.4 \%)$, which could be attributed to the introduction of $-\mathrm{SO}_{3}{ }^{-}$ groups and the thermal decomposition of side chains on the alginate chains. Additionally, the weight retention ratio of HepMBm exceeded that of SA within a wide range of temperature, which indicated that the thermal stability of HepMBm increased. The DTG curves of these biomacromolecules are shown in Figure S3 (B), and the peaks of degradation ratio at $89{ }^{\circ} \mathrm{C}$ and $252{ }^{\circ} \mathrm{C}$ were observed for both SA and HepMBm, which was consistent with the two similar decomposition stages of SA and HepMBm. There was a new peak at $717{ }^{\circ} \mathrm{C}$ in the DTG curve of HepMBm, which might result from the decomposition of $-\mathrm{SO}_{3}{ }^{-}$groups or the residual DCC.
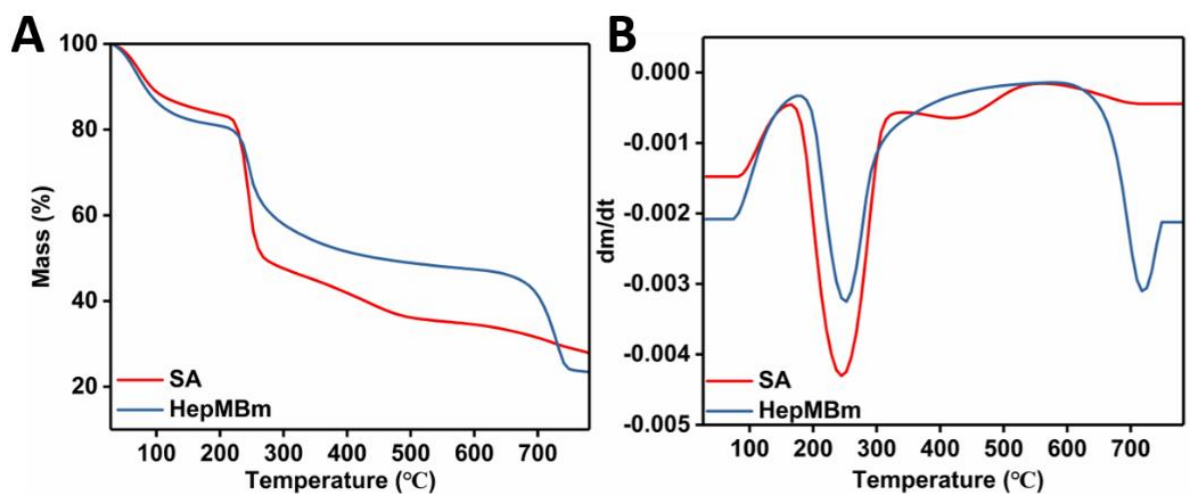

Figure S3. TGA curves (A) and DTG curves (B) for SA and HepMBm. 
The toxicity, immunogenicity and stability of HepMBm were also investigated in case it is released to the blood stream. Since blood cells are the cells that HepMBm is mostly likely to contact, a systematic blood count assay in vitro was performed to study the effect of HepMBm on the volume distribution of hemocyte and study the effect on blood cells further. The differential white blood cell count (DIFF) scatter charts of normal whole blood (up) and whole blood after incubating with HepMBm are shown in Figure S4 (A). The morphology and size distribution of the five types of white blood cells (neutrophil granulocyte, eosinophilic granulocyte, basophilic granulocyte, lymphocyte and monocyte) were not affected by HepMBm, indicating the negligible effect of the macromolecule on white blood cells. As shown in Figure S4 (B), HepMBm did not cause severe distinct changes in the numbers of various blood cells, demonstrating its low toxicity. Besides, as shown in and Figure S4 (C), severe distinct changes in the volume distribution of blood cells were also not observed by HepMBm. These results indicated that the macromolecule could be used to treat whole blood safely. Figure S4 (D) showed the immunogenicity of HepMBm. Comparing with normal whole blood, the blood after incubating with HepMBm showed lower activation level of different activation system, indicating the low immunogenicity of HepMBm. As shown in Figure S4 (E), after incubating the nanocomposite spheres with PBS, the rinse solution showed no difference comparing with pristine PBS, which indicated that no macromolecule was eluted, thus demonstrating the high stability of the conjugation. 

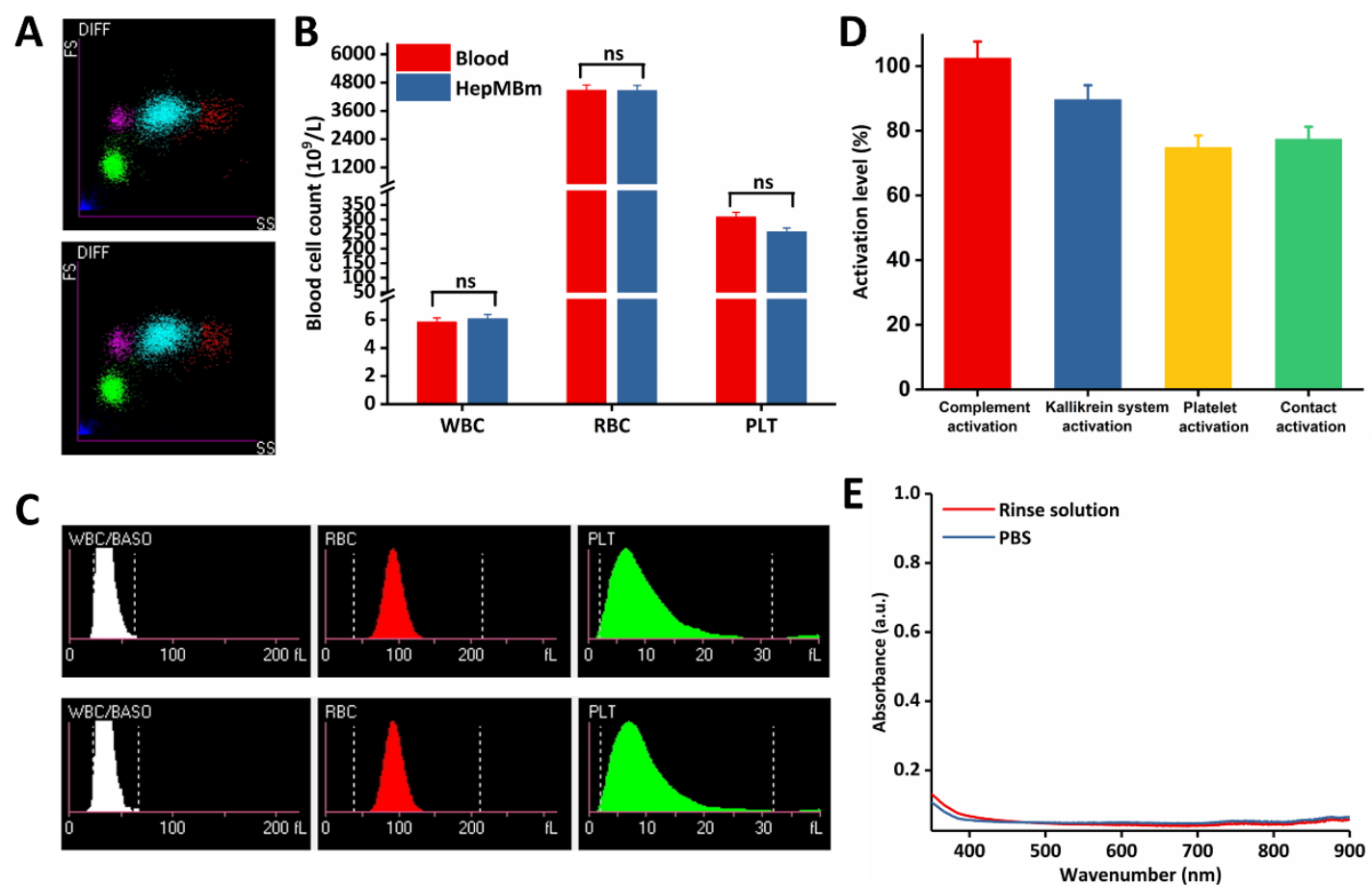

Figure S4. (A) The differential white blood cell count (DIFF) scatter charts of whole blood (up) and the blood after incubating with HepMBm (down). (B) Comparison of the blood cell count for normal whole blood and blood after incubating with HepMBm. (C) The white blood cell, red blood cell and platelet volume distribution curves of normal whole blood (up) and the blood after incubating with HepMBm (down). (D) The activation level (complement activation level, kallikrein system activation level, platelet activation level and contact activation level) of the blood after incubating with HepMBm. (E) The UV spectra for pristine PBS and the solution after incubating with nanocomposite spheres. 

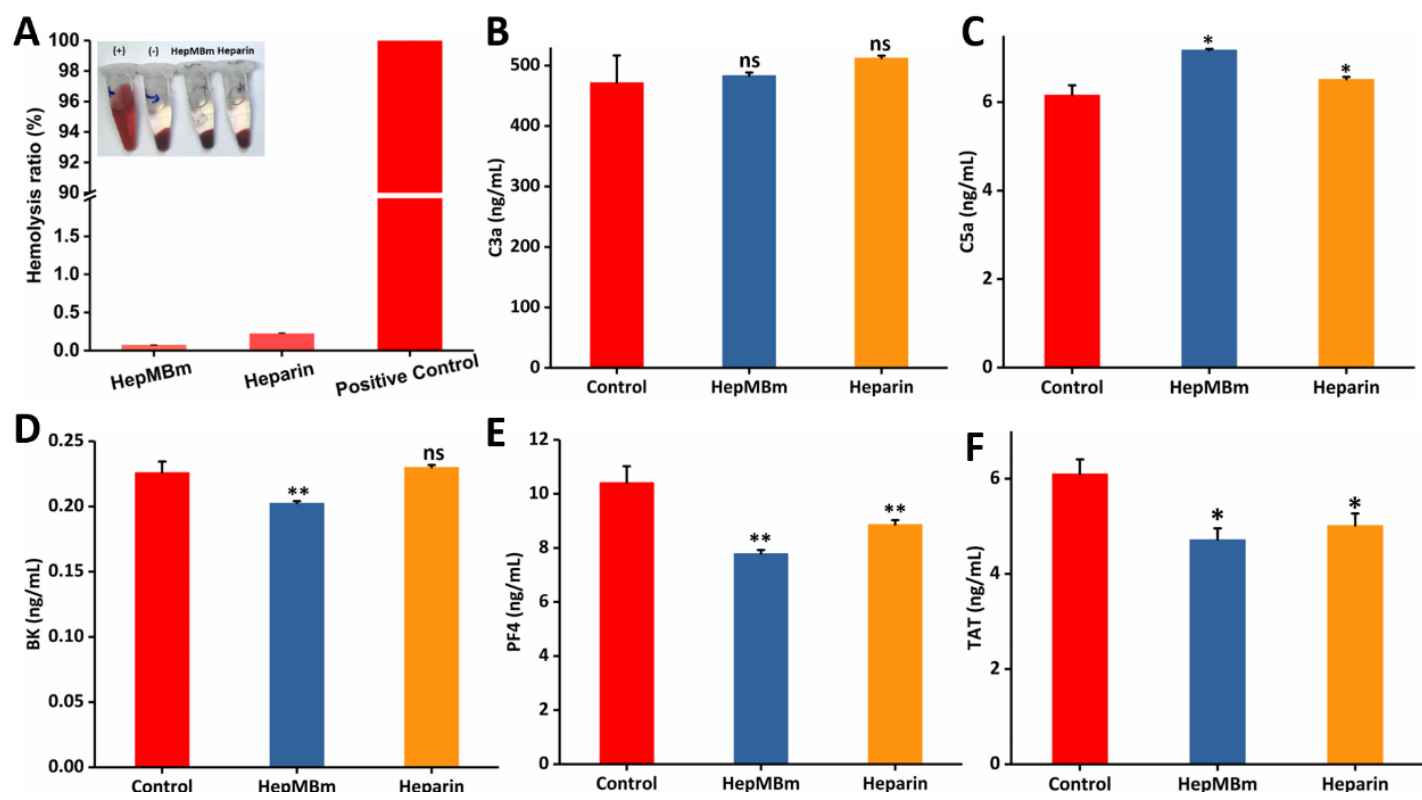

Figure S5. (A) The hemolysis ratios of HepMBm and heparin incubated with RBCs at $37{ }^{\circ} \mathrm{C}$ for $3 \mathrm{~h}$ with shaking. The red solution indicates that the RBCs were destroyed and hemoglobin was released. (-) represents negative control and (+) represents positive control. The generated concentrations of C3a (B), C5a (C), BK (D), PF4 (E) and TAT (F) after incubating the HepMBm and heparin with whole blood. 
Table S1. The physical dimension of the four kinds of nanocomposite spheres. $(n=12)$

\begin{tabular}{cc}
\hline Sample & Physical Dimension $(\mathrm{mm})$ \\
\hline rGO@HepMBm0 & $1.27 \pm 0.33$ \\
rGO@HepMBm1 & $1.15 \pm 0.21$ \\
rGO@HepMBm2 & $1.43 \pm 0.22$ \\
rGO@HepMBm3 & $1.22 \pm 0.15$ \\
\hline
\end{tabular}


The surface micro-morphologies of the spheres were characterized by SEM images. As shown in Figure S6, the wrinkle-like surface of the spheres could be observed, suggesting the successful introduction of rGO. Compared to rGO@HepMBm0 and rGO@HepMBm1, more dense and smooth surface morphologies of rGO@HepMBm2 and rGO@HepMBm3 were observed. The reasons for the changes might be as follows: (i) The increased viscosity and density by introducing the HepMBm made it more difficult for the oligomer to be eluted during the washing process. Hence, the uniform surface morphologies could be remained. (ii) The long chain molecules of the HepMBm could warp around the rGO, and thus the dispersity of rGO in the spheres was better with the introduction of HepMBm.

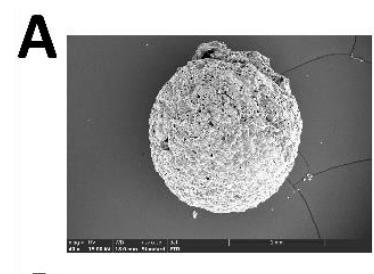

C

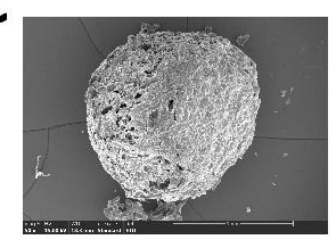

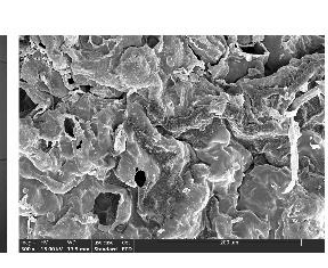

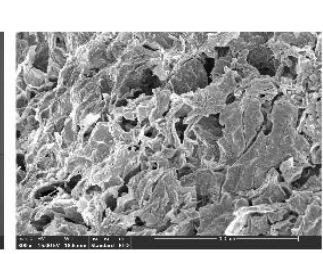

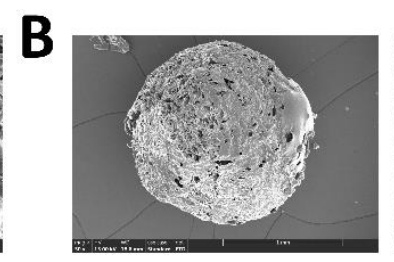

D

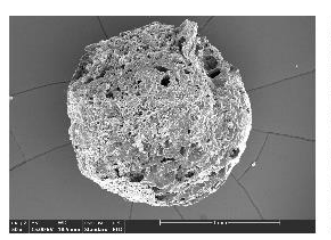

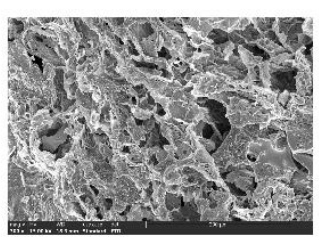

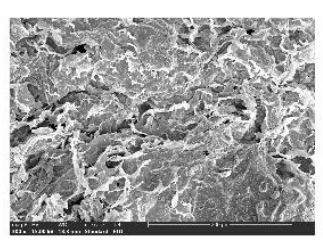

Figure S6. The surface morphologies of the nanocomposite spheres (magnification from left to right: $\times 40$ and ×300). (A) rGO@HepMBm0. (B) rGO@HepMBm1. (C) rGO@HepMBm2. (D) rGO@HepMBm3. 


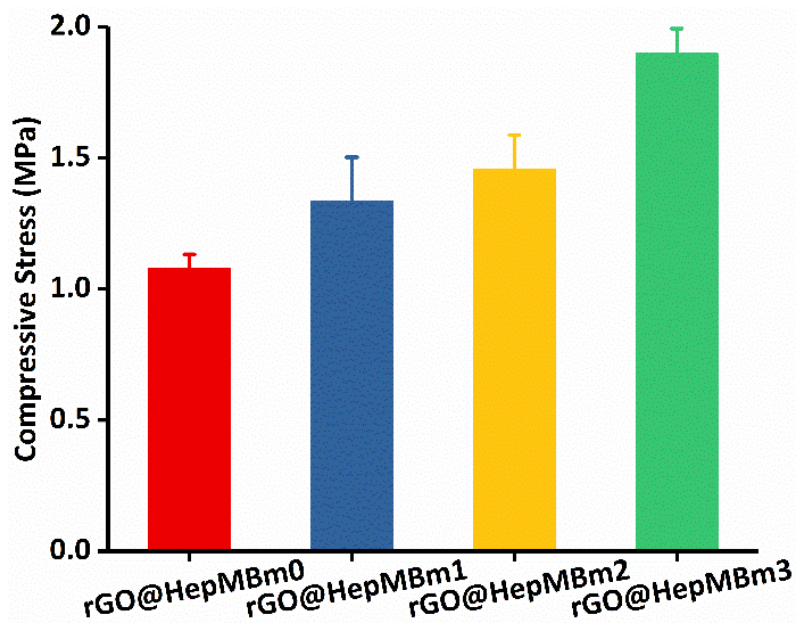

Figure S7. The compressive stress of the rGO@HepMBm0, rGO@HepMBm1, rGO@HepMBm2 and rGO@HepMBm3 nanocomposite spheres. 


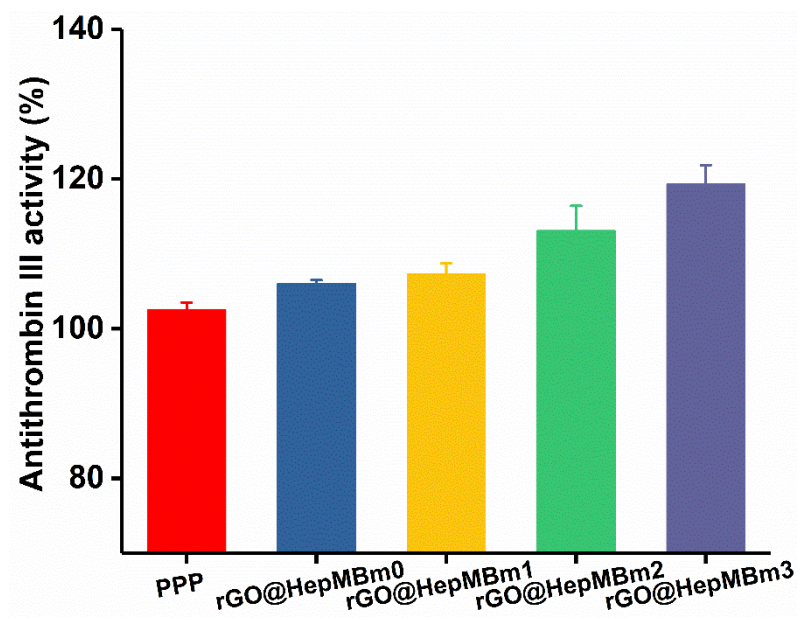

Figure S8. The antithrombin III activity for PPP after incubation with different nanocomposite spheres. 
To further show the role of introducing pore-foaming agent $\mathrm{CaCO}_{3}$ which can improve the bilirubin adsorption activity and dimensional stability of spheres, nanocomposite spheres HepMBmX $(\mathrm{X}=0,1,2,3)$ without calcium (named as no CaHepMBmX) and SA spheres were prepared, and their adsorption capability towards bilirubin and dimensional stability were systematically investigated to show comparison. As shown in Figure S9 (A), SA spheres showed low adsorption amount for bilirubin because they lack effective interaction with bilirubin such as $\pi-\pi$ interaction or electrostatic interaction, while the rGO-based nanocomposite spheres all showed relatively high adsorption capability (higher than $200 \mathrm{mg} / \mathrm{g}$ ). Comparing with the nanocomposite spheres without calcium, the nanocomposite spheres with calcium showed obviously higher adsorption activities because the introduction of porefoaming agent can provide more pathways for the transport of bilirubin and improve the adsorption capability of the spheres. Figure S9 (B) showed the swelling ratios of the spheres. SA spheres showed ultrahigh swelling ratios due to their hydrophilia, which can reach about $43 \mathrm{~g} / \mathrm{g}$. The high swelling seriously restricts their potential applications where a high flow rate is used. For the rGO-based spheres, the spheres with calcium showed lower water uptake because the swellings of the spheres were restricted by increasing degree of cross-linking of polymer network and the dual-network structure of the beads, indicating that the nanocomposite spheres with calcium could possess high size-stability during the hemoperfusion treatment. 

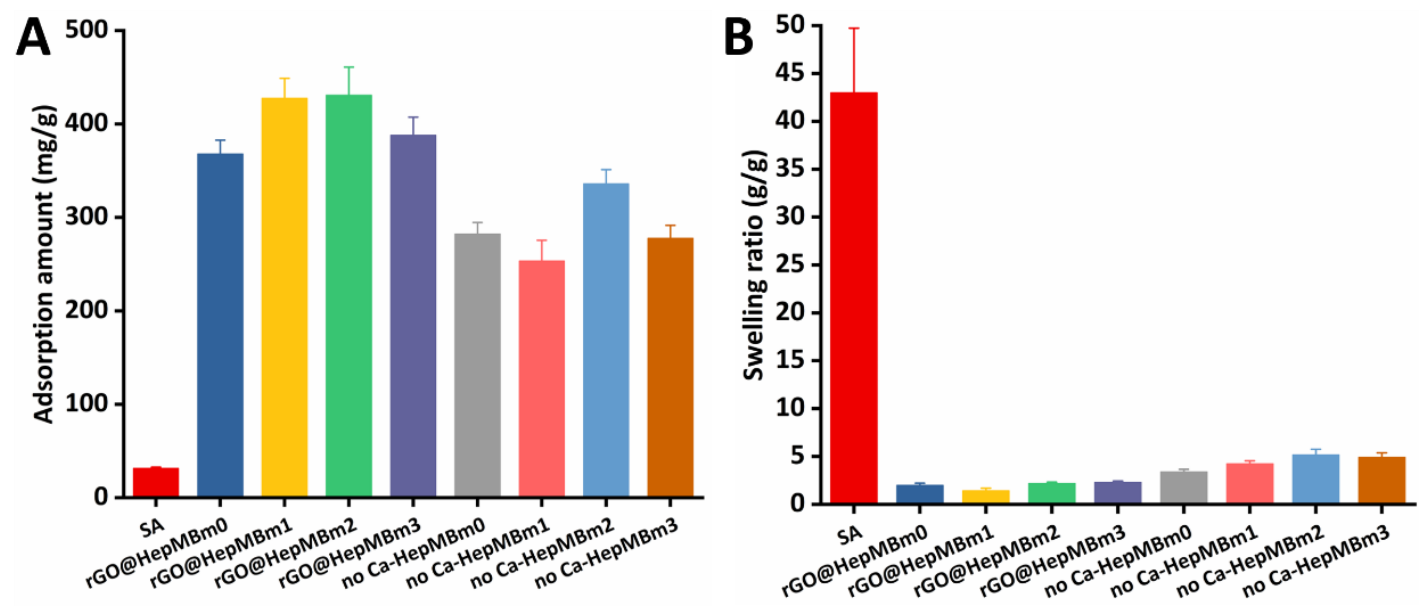

Figure S9. (A) The bilirubin adsorption amounts of SA spheres, nanocomposite spheres with calcium (named as rGO@HepMBmX) and nanocomposite spheres without calcium (named as no Ca-HepMBmX). (B) The swelling ratios of SA spheres, nanocomposite spheres with calcium (named as rGO@HepMBmX) and nanocomposite spheres without calcium (named as no Ca-HepMBmX). 


\section{Adsorption isotherm studies}

The adsorption isotherms describe the interaction between absorbent and absorbate, and they are critical for optimizing the application of absorbents. Herein, Langmuir isotherm and Freundlich isotherm are applied, since they are two of the most widely used adsorption isotherms to study the adsorption of solute from liquid solutions. The Langmuir model assumes that all adsorption sites are homogeneous, the adsorption process is a dynamic balance, the adsorption occurs in monolayer, and the adsorbed molecules are all independent. ${ }^{10,11}$ It can be expressed as follows:

$$
\frac{C_{e}}{q_{e}}=\frac{1}{q_{\max } k_{L}}+\frac{C_{e}}{q_{\max }}
$$

where $C_{e}(\mathrm{mg} / \mathrm{L})$ is the concentration of the bilirubin at equilibrium; $q_{e}(\mathrm{mg} / \mathrm{g})$ is the amount of bilirubin adsorbed by the unit mass after the adsorption reaches equilibrium; $q_{\max }(\mathrm{mg} / \mathrm{g})$ is the adsorption capacity; $K_{L}(\mathrm{~L} / \mathrm{mg})$ is the Langmuir adsorption constant.

Freundlich isotherm is an empirical equation used to describe the heterogeneous system and it depicts reversible adsorption. It can be expressed by the following equation: ${ }^{12}$

$q_{e}=k_{F} C_{e}^{1 / n}$

where $1 / n$ is the constant which incorporate factors affecting the adsorbed amount at equilibrium; $k_{F}$ is the Freundlich isotherm constant $(\mathrm{mg} / \mathrm{g})(\mathrm{L} / \mathrm{mg})^{1 / \mathrm{n}}$; the meanings of $q_{e}(\mathrm{mg} / \mathrm{g})$ and $C_{e}(\mathrm{mg} / \mathrm{L})$ are the same as those in the Langmuir equation.

The linear form of the Freundlich isotherm equation is as follows:

$$
\ln q_{e}=k_{F}+(1 / n) \ln C_{e}
$$



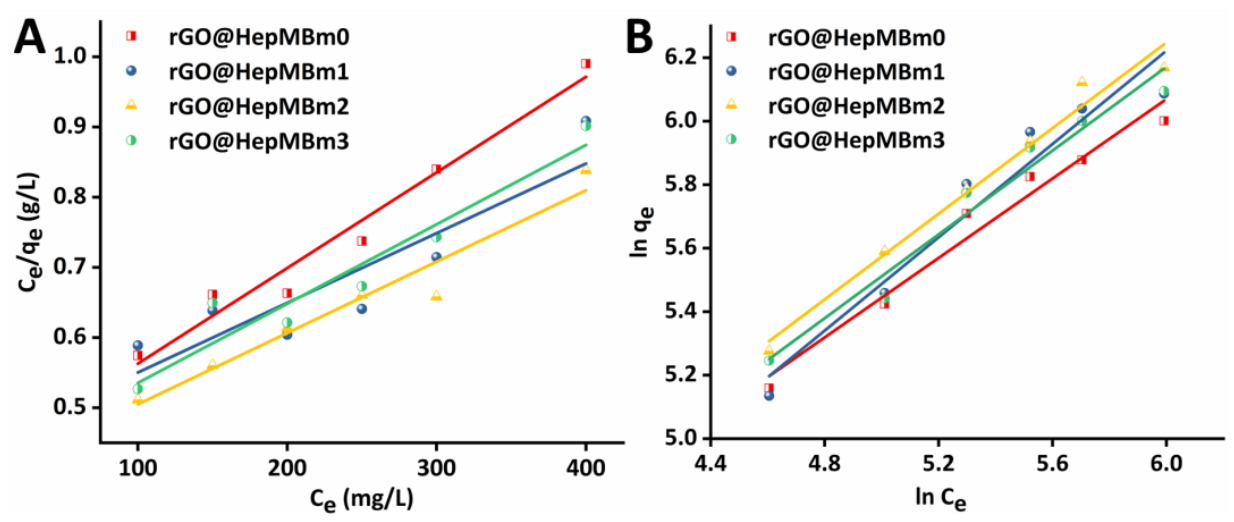

Figure S10. (A) Langmuir isotherm model. (B) Freundlich isotherm model. 


\section{Adsorption kinetic studies}

Furthermore, pseudo-first-order, pseudo-second-order and intraparticle diffusion kinetic models were employed to analyze the bilirubin adsorption process. The pseudofirst-order equation is the first-rate equation for the adsorption of liquid-solid system based on solid capacity ${ }^{13}$ and can be expressed by the following equation: ${ }^{14}$

$\ln \left(q_{e}-q_{t}\right)=\ln q_{e}-k_{1} t$

where $\mathrm{k}_{1}$ is the rate constant of pseudo-first-order kinetic model; $\mathrm{q}_{\mathrm{t}}$ is the adsorption amount of bilirubin at time $\mathrm{t}(\mathrm{mg} / \mathrm{g})$; $\mathrm{q}_{\mathrm{e}}$ is that adsorbed at the equilibrium $(\mathrm{mg} / \mathrm{g})$.

For the pseudo-second-order reaction, the rate depends on the adsorbed amount when the adsorption reached equilibrium and the adsorbed amount on the surface of adsorption material. ${ }^{15}$ The equation can be written as follows: ${ }^{14}$

$$
\frac{t}{q_{t}}=\frac{1}{k_{2} q_{e}^{2}}+\frac{t}{q_{e}}
$$

where $\mathrm{k}_{2}$ is the rate constant of the pseudo-second-order kinetic model; the meaning of $\mathrm{q}_{\mathrm{t}}, \mathrm{q}_{\mathrm{e}}$, and $\mathrm{t}$ are the same as those in Eq. (S7).

The intra-particle diffusion model is also used to identify the diffusion mechanism and it can be represented by the following equation: ${ }^{16}$

$q_{t}=k_{p} t^{1 / 2}+C$

where $k_{p}\left(\mathrm{mg} / \mathrm{g} \min ^{1 / 2}\right)$ and $C(\mathrm{mg} / \mathrm{g})$ are the constant of intraparticle diffusion model; the meaning of $\mathrm{q}_{\mathrm{t}}$ is the same as that in Eq. (S7). 

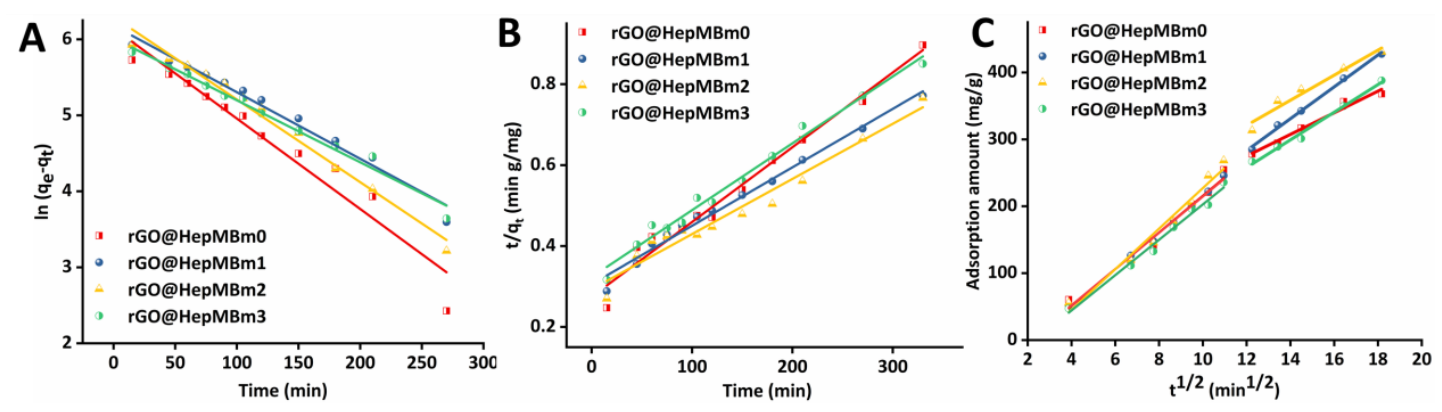

Figure S11. (A) Application of the pseudo-first-order adsorption model for the bilirubin adsorption onto the spheres. (B) Application of the pseudo-second-order adsorption model for the bilirubin adsorption onto the spheres. (C) Application of the intraparticle diffusion model for the bilirubin adsorption onto the spheres. 
Table S2. The pseudo-first-order kinetic models and the parameters for the adsorption of bilirubin to the spheres.

\begin{tabular}{ccccc}
\hline \multirow{2}{*}{$\begin{array}{c}\text { Parameter } \\
\mathrm{s}\end{array}$} & $\begin{array}{c}\text { rGO@HepMBm } \\
0\end{array}$ & rGO@HepMBm & rGO@HepMBm & rGO@HepMBm \\
\hline $\begin{array}{c}\mathrm{K}_{1}\left(\mathrm{~min}^{-1}\right) \\
\mathrm{Q}_{\mathrm{e}, \mathrm{cal}}\end{array}$ & 0.01189 & 0.00876 & 0.01087 & 0.00816 \\
$(\mathrm{mg} / \mathrm{g})$ & 467.2993 & 483.2383 & 542.1811 & 409.3825 \\
$\mathrm{R}^{2}$ & 0.93490 & 0.97499 & 0.98303 & 0.98121 \\
\hline
\end{tabular}


Table S3. The pseudo-second-order kinetic models and the parameters for the adsorption of bilirubin to the spheres.

\begin{tabular}{|c|c|c|c|c|}
\hline \multirow{3}{*}{$\begin{array}{c}\text { Parameter } \\
\text { s }\end{array}$} & \multicolumn{4}{|c|}{ Samples } \\
\hline & rGO@HepMBm & rGO@HepMBm & rGO@HepMBm & rGO@HepMBm \\
\hline & 0 & 1 & 2 & 3 \\
\hline $\begin{array}{c}\mathrm{Q}_{\mathrm{e}, \mathrm{cal}} \\
(\mathrm{mg} / \mathrm{g})\end{array}$ & 540.5405 & 689.6552 & 735.2941 & 606.0606 \\
\hline $\begin{array}{c}\mathrm{K}_{2}\left(\mathrm{~g} \mathrm{mg}^{-}\right. \\
\left.{ }^{1} \mathrm{~min}^{-1}\right)\end{array}$ & $1.2459 \mathrm{E}-05$ & $6.88058 \mathrm{E}-06$ & 6.30596Е-06 & $8.41655 \mathrm{E}-06$ \\
\hline $\mathrm{R}^{2}$ & 0.97882 & 0.98625 & 0.96009 & 0.98598 \\
\hline
\end{tabular}


Table S4. The intra-particle diffusion kinetic models and the parameters for the adsorption of bilirubin to the spheres.

\begin{tabular}{cccccc}
\hline \multirow{2}{*}{$\begin{array}{c}\text { Parameter } \\
\text { s }\end{array}$} & rGO@HepMBm & rGO@HepMBm & rGO@HepMBm & rGO@ HepMBm \\
& & 0 & 1 & 2 & 3 \\
\hline \multirow{2}{*}{ Step } & $\mathrm{C}_{1}$ & -58.55325 & -56.25726 & -75.93639 & -61.37493 \\
1 & $\mathrm{k}_{\mathrm{p} 1}$ & 27.43302 & 27.09343 & 30.32326 & 26.42735 \\
& $\mathrm{R}_{1}{ }^{2}$ & 0.97657 & 0.99655 & 0.97460 & 0.98651 \\
\hline \multirow{2}{*}{ Step } & $\mathrm{C}_{2}$ & 81.15143 & -2.31084 & 96.29406 & 10.16489 \\
2 & $\mathrm{k}_{\mathrm{p} 2}$ & 16.16785 & 23.80516 & 18.73196 & 20.65547 \\
& $\mathrm{R}_{2}{ }^{2}$ & 0.97323 & 0.99629 & 0.96000 & 0.99065 \\
\hline
\end{tabular}

\section{Reference}

1. Hummers, W. S.; Offeman, R. E., PREPARATION OF GRAPHITIC OXIDE. J. Am. Chem. Soc. 1958, 80, 1339-1339.

2. Zhao, W.; Glavas, L.; Odelius, K.; Edlund, U.; Albertsson, A.-C., A robust pathway to electrically conductive hemicellulose hydrogels with high and controllable swelling behavior. Polymer 2014, 55, 2967-2976.

3. Bonnett, R., Mechanisms of the photodegradation of bilirubin Biochem. Soc. Trans. 1976, 4, 222-226.

4. Ma, L.; Cheng, C.; Nie, C.; He, C.; Deng, J.; Wang, L.; Xia, Y.; Zhao, C., Anticoagulant sodium alginate sulfates and their mussel-inspired heparin-mimetic coatings. J. Mater. Chem. B 2016, 4, 3203-3215.

5. Huang, R. H.; Du, Y. M.; Yang, J. H., Preparation and in vitro anticoagulant activities of alginate sulfate and its quaterized derivatives. Carbohydr. Polym. 2003, 52, 19-24. 
6. Freeman, I.; Kedem, A.; Cohen, S., The effect of sulfation of alginate hydrogels on the specific binding and controlled release of heparin-binding proteins. Biomaterials 2008, 29, 3260-3268.

7. Wang, H. M.; Li, H. X.; Yu, X. Y.; Ren, Z. G.; Lang, J. P., Cyclodimerization and cyclotrimerization of isocyanates promoted by one praseodymium benzenethiolate complex $\left[\operatorname{Pr}(\mathrm{SPh})_{3}(\mathrm{THF})_{3}\right]$. Tetrahedron 2011, 67, 1530-1535.

8. Wu, X.; Niu, Q.; Li, T., Novel one-pot synthesis of symmetrically substituted ureas. Synth. Commun. 2016, 46, 1364-1369.

9. Monsur, H. A.; Jaswir, I.; Simsek, S.; Amid, A.; Alam, Z., Chemical structure of sulfated polysaccharides from brown seaweed (Turbinaria turbinata). Int. J. Food Prop. 2016, 20, 1457-1469.

10. Namasivayam, C.; Kavitha, D., Removal of Congo Red from water by adsorption onto activated carbon prepared from coir pith, an agricultural solid waste. Dyes Pigment. 2002, 54, 47-58.

11. Yao, Y.; Xu, F.; Chen, M.; Xu, Z.; Zhu, Z., Adsorption behavior of methylene blue on carbon nanotubes. Bioresour. Technol. 2010, 101, 3040-3046.

12. Xia, B. L.; Zhang, G. L.; Zhang, F. B., Bilirubin removal by Cibacron Blue F3GA attached nylon-based hydrophilic affinity membrane. J. Membr. Sci. 2003, 226, 9-20.

13. Ho, Y. S.; McKay, G., The sorption of lead (II) ions on peat. Water Res. 1999, 33, $578-584$. 
14. Sen Gupta, S.; Bhattacharyya, K. G., Removal of Cd (II) from aqueous solution by kaolinite, montmorillonite and their poly (oxo zirconium) and tetrabutylammonium derivatives. J. Hazard. Mater. 2006, 128, 247-257.

15. Dogan, M.; Ozdemir, Y.; Alkan, M., Adsorption kinetics and mechanism of cationic methyl violet and methylene blue dyes onto sepiolite. Dyes Pigment. 2007, 75, 701713.

16. Yang, X. Y.; Al-Duri, B., Kinetic modeling of liquid-phase adsorption of reactive dyes on activated carbon. J. Colloid Interface Sci. 2005, 287, 25-34. 